\section{$\underset{\substack{\text { hommes } \\ \text { \& migrations }}}{ }$}

\section{Hommes \& migrations}

Revue française de référence sur les dynamiques

migratoires

1329 | 2020

Migrations et création littéraire

\title{
Migration et création littéraire
}

\section{Odile Richard - Pauchet}

\section{CpenEdition \\ Journals}

\section{Édition électronique}

URL : https://journals.openedition.org/hommesmigrations/11054

DOI : 10.4000/hommesmigrations. 11054

ISSN : 2262-3353

\section{Éditeur}

Musée national de l'histoire de l'immigration

\section{Édition imprimée}

Date de publication : 1 avril 2020

Pagination : 9-11

ISBN : ISBN 978-2-919040-50-6

ISSN : $1142-852 X$

\section{Référence électronique}

Odile Richard - Pauchet, « Migration et création littéraire », Hommes \& migrations [En ligne], 1329 | 2020, mis en ligne le 01 avril 2020, consulté le 03 janvier 2023. URL : http://journals.openedition.org/ hommesmigrations/11054; DOI : https://doi.org/10.4000/hommesmigrations.11054 


\section{Migration \\ et création littéraire}

Odile Richard-Pauchet,

maître de conférences HDR en littérature française, université de Limoges,

Faculté des lettres et des sciences humaines (FLSH).

$\underline{\mathrm{A}}$

l'âge classique, voici comment l'on définissait, dans l'Encyclopédie de Diderot, la notion d'exil: «Chez les Romains le mot exil, exilium, signifiait proprement une interdiction, ou exclusion de l'eau \& du feu, dont la conséquence naturelle était, que la personne ainsi condamnée était obligée d'aller vivre dans un autre pays, ne pouvant se passer de ces deux éléments [...]. Ce mot est dérivé du mot latin exilium ou de exul, qui signifie exilé; \& les mots exilium ou exul sont formés probablement d'extra solum, hors de son pays natal'.»

Hier, l'exil ou l'ostracisme étaient d'abord une exclusion ou une expulsion politique, seule raison humaine de s'expatrier, tant était fort l'attachement au sol. Aujourd'hui, c'est parce que la personne est démunie de tout (d'eau, de feu, mais aussi de liberté, de travail) qu'elle se résout à la migration. Cette exclusion vis-à-vis de l'essentiel entraîne une aspiration très forte à retrouver cette eau et ce feu, synonymes de vie, de nourriture, de foyer d'une part, mais aussi de création ou de possibilité de création d'autre part, car la création, comme le rire, est le propre de l'homme. Prométhée n'a-t-il pas volé le feu aux Dieux afin d'affirmer son humanité? L'eau et le feu sont d'ailleurs des éléments que l'on retrouve partout dans la création: l'eau pour diluer sa peinture, pour laver ses pinceaux; le feu pour faire cuire sa céramique (et pas seulement à Limoges), pour chanter même - "Allumer le feu», comme le dit un certain chanteur. L'écriture, la poésie sont une chaleur qui s'élève entre les hommes. Elles coulent comme une eau désaltérante dans la gorge de ceux qui ont soif: «Eaux coulez coulez allez allez vers la mer./ Lave le sel toute eau répandue toute eau repentie. » (Léopold Sédar Senghor, «Élégie des eaux», in Nocturnes, 1961). «Feu que les hommes regardent dans la nuit, dans la nuit profonde,/ Feu qui brûles et ne chauffes pas, qui brilles et ne brûles pas, / Feu qui voles sans corps et sans cœur, qui ne connais case ni foyer... " (Léopold Sédar Senghor, "Chant du feu», in Poèmes, 1964).

Le 9 avril 2019, nous avons souhaité consacrer une journée d'étude, avec les étudiants du master Lettres et arts de l'université de Limoges, à l'analyse des liens privilégiés qui unissent la migration à la création ${ }^{2}$. Soit parce que l'art est une activité, comme nous l'avons dit, consubstantielle au déplacement, à la fuite, à l'exil, parce que l'action même de reconstituer un foyer quand on l'a perdu, relève de l'acte de création: partir du néant pour retrouver un tout. Soit aussi parce que cette activité créatrice (d'écriture, de peinture, de musique...) aide à supporter l'exil par la chaleur humaine qu'elle dégage et les nouveaux liens qu'elle permet de développer. Soit, enfin, parce qu'elle est elle-même une interrogation sur cet événement d'exil, étant donné que tout acte créateur exige une forme d'aliénation à soi-même. S'exiler dans la

\footnotetext{
1. http://enccre.academie-sciences.fr/encyclopedie/article/ v6-335-0/.

2. Appel à communication sur : https://www.unilim.fr/ recherche/2019/02/14/migration-et-creation/, programme définitif sur : https://www.flsh.unilim.fr/wp-content/uploads/ sites/9/2019/03/Programme-migration-et-creation.pdf.
} 
création, c'est peut-être donc se trouver un peu mieux en accord avec soi-même, lorsqu'on est exilé géographique et déjà devenu «autre» à soi-même, et aux autres.

Les hommes et les femmes qui ont répondu présent à notre invitation du 9 avril dernier sont d'abord des passionnés et des connaisseurs de la question de la migration: Jean-Michel Devésa, professeur de littérature française et d'études francophones, directeur du master de Limoges «Textes et représentations du monde» (TRM); Alexis Nouss, professeur de littérature et de littérature comparée à l'université d'Aix-Marseille, spécialiste reconnu de la question migratoire; Marie Virolle, éditrice de la revue Marsa - Revue A, férue de questions algériennes; Marie Poinsot, rédactrice en chef de la revue Hommes \& Migrations; un plasticien, Stéphane Lhomme qui a illustré ce numéro; de jeunes universitaires, qui se sont exprimées via des enquêtes sur des écrivains de l'émigration dont elles sont spécialistes; enfin, des artistes, comme le poète Adama Diané3, journaliste guinéen lui-même réfugié politique, étudiant inscrit à l'université de Limoges, et le chanteur Mamadi, migrant, ont contribué à animer cette journée. Ont enfin répondu à notre appel un public d'étudiants mais aussi d'exilés et de migrants qui ont été nos voisins pendant quelques mois, squattant un bâtiment désaffecté du campus, et qui ont eu la délicatesse de nous y accueillir pour partager un déjeuner le 9 avril 2019.

Marie Poinsot nous a proposé de contribuer à ce dossier d'avril 2020 de la revue Hommes \& Migrations, invitant par la suite à nous y rejoindre d'autres auteur.e.s sensibles à notre thématique. Nous esquisserons rapidement les quelques problématiques qui traversent cet ensemble.

Avec Karla Segura Pantoja de l'université de Cercy-Pontoise, nous avons initialement souhaité mettre en valeur la migration datant de la Seconde Guerre mondiale, des surréalistes exilés au Mexique, qui constitue l'une des premières étapes, au XXe siècle, d'un dialogue artistique « Nord-Sud», peut-être mieux connu jusqu'ici à travers les voyages publiés de Michel Leiris en Afrique (L'Afrique fantôme, 1934) et de Claude Levi-Strauss en Amazonie (Tristes Tropiques, 1955).

Ce «dialogue» fut toutefois fragile et non exempt d'ambiguïtés très fortes. Jean-Michel Devésa, dans un entretien avec $H \& M$, mais également Kaoutar Harchi, dans son étude «Paris comme condition? Une approche spatialisée de la valorisation artistique et culturelle», rappellent comme il est, depuis la période de la décolonisation, d'une extrême complexité pour un écrivain issu des anciennes colonies de se faire entendre, comprendre, éditer, reconnaître dans le pays et la langue de l'ancien colon, notamment le français.

Ainsi, à titre d'exemple, nous verrons comment en Europe, après les étapes de colonisation, puis de décolonisation effectuées dans la violence (par la fracture que constitue la guerre d'Algérie, mais pas seulement), des auteur.e.s chassé.e.s, ou issu.e.s de familles chassées par différents conflits, peinent à trouver la langue, le lieu et la forme d'accueil institutionnel adéquats pour dire le traumatisme. Charikleia Magdalini Kefalidou (université Paris-Sorbonne) évoque ainsi, avec «L'écrilibriste» sur Denis Donikian, la tentative presque impossible pour un écrivain d'origine arménienne de rendre compte du génocide dans la langue de l'exil.

Le journaliste Mustapha Harzoune, dans un tour d'horizon plus vaste de la littérature de langue française, montre comment "corps, sexualités et métissages romanesques» sont une des thématiques majeures de cette littérature d'émigration, lorsqu'elle parvient à franchir ce barrage socio-culturel, socio-poétique et socio-politique qu'est le milieu parisien de l'édition. Ce dernier donne lui-même la parole, dans un autre entretien, à l'écrivaine Tassadit Imache afin de rendre compte de l'expérience

3. Certains articles n'ont pas été publiés dans ce numéro pour des raisons de calendrier éditorial. C'est le cas de l'entretien réalisé par Eugénie Péron-Douté (université de Limoges) avec Omid Dashti, metteur en scène iranien arrivé à Bruxelles en 2000, qui exprime les mêmes interrogations et les mêmes doutes quant à la validité de la langue française pour dire le « Je » iranien resté en souffrance, y compris dans ses entreprises théâtrales les plus inventives. Sarra Boukhatem, de l'université de Mostaganem (Algérie), décrit cette « écriture de l'entre-deux dans Du rêve pour les oufs de Faiza Guène » dans un article issus de sa communication au colloque. L'une des belles illustrations également du parcours de migration, dans sa cruauté, voire sa dimension moraliste ou immoraliste, revient à Sylvie Coly (University of the Gambia), qui compare méthodiquement, grâce à deux romans anglophones contemporains issus de ce pays, l'échec ou le succès du terrible périple de la « Mort-

Méditerranée » promis aux jeunes Gambiens. Ces articles feront l'objet d'une publication ultérieure dans la revue Hommes \& Migrations. Adame Diané n'a hélas pas répondu à notre sollicitation de publier certains de ses poèmes lus le 9 avril 2019. II avait particulièrement ému et retenu l'attention des étudiants et du public limougeaud en écrivant des poèmes sur des paquets de cigarettes, seul support dont il pouvait disposer lors de son exil. Voir https://france3-regions.francetvinfo.fr/nouvelleaquitaine/haute-vienne/limoges/journaliste-guineen-adamadiane-peut-faire-sa-demande-asile-france-1495479.html. 
éprouvante qui consiste à percer ce milieu pour imposer une vision authentique de son métissage ou de son altérité («La pluralité des visages de la France est une réalité»).

Alexis Nouss, intervenu lors de la journée d'étude pour faire remonter à notre mémoire quelques archétypes, tels Ulysse ou du Bellay, des grandes figures exiliques, propose dans ce dossier un vaste parcours européen des auteur.e.s de l'exil, suivi d'une enquête le long d'une mémoire familiale: celle de l'œuvre de Omar Benlaala, Tu n'habiteras jamais Paris, qui se présente comme un livre à deux voix faisant alterner la parole d'un maçon, Bouzid Benlaala, immigré kabyle des années 1960, et la parole d'un paysan creusois devenu soldat puis maçon puis député, Martin Nadaud (1815-1898). Cette alternance très forte d'exilés obscurs devenus célèbres, avec d'autres obscurs qui le deviendront peut-être un jour, met en perspective et en relativité tout le processus du parcours exilique, rendant dérisoire et pitoyable toute tentative de mépriser ou de condamner le migrant pour cela seul qui fait sa force aujourd'hui, et peutêtre sa notoriété de demain.

Ce tour d'horizon de la parole de l'altérité rapporté à notre univers métropolitain, trop souvent spectateur, consommateur, juge et partie, serait nécessairement incomplet s'il ne continuait d'interroger d'autres communautés inaudibles comme telles, parce qu'invisibles d'un point de vue matériel et institutionnel. Ewa Tartakowsky présente ainsi ces «Lieux institutionnels, territoires symboliques: les espaces de rencontre pour les écrivains d'origine judéo-maghrébine en France». Tandis que Simon Guy, artiste et musicien, enquêteur à l'Agence des musiques des territoires d'Auvergne (AMTA), rend compte d'une démarche de terrain consistant à aller enregistrer les voix et les sons du quartier Saint-Jacques de ClermontFerrand afin de constituer un "atlas sonore» de ce monde bientôt oublié, conservé ici sous la forme itinérante d'une géographie immatérielle.

Nos vifs remerciements vont d'abord aux étudiant.e.s, initiateur.trices et organisateur.trices de ce projet; à Marie Poinsot, pour son accueil et son hospitalité éditoriale faite à ces «paroles migrantes»; enfin à tous les auteur.e.s de ces textes qui ont participé les premier.e.s, à cette belle aventure, ou bien l'ont rejointe en cours de route, à différentes étapes de son itinéraire, comme pour mieux rendre sensible ce cheminement qu'il est un devoir d'effectuer auprès de celles et de ceux dont on rend compte ici des traces, des marches, des espoirs, des navigations, des renoncements, des accomplissements, des aboutissements, sur la voie d'une autre et toujours impossible patrie. 